\title{
Region-Based Segmentation of Textured Images
}

\author{
Catherine Rouquet and Pierre Bonton
}

LAboratoire des Sciences et Matériaux pour l'Electronique, et d'Automatique -URA 1793 CNRS- Université B. Pascal F-63177 Aubière Cedex

\begin{abstract}
This paper presents a region-based segmentation algorithm which can be applied to various problems since it does not require a priori knowledge concerning the kind of processed images. This algorithm, based on a split and merge method, gives results both on homogeneous grey level images and on textured images. We modeled exploited fields by Markov Random Fields (MRF), the segmentation is then optimally determined using the Iterated Conditional Modes (ICM). Results from road scenes without white lines are presented.
\end{abstract}

\section{Introduction}

The creation of an autonomous vehicle has been the subject of various research programs. To answer this question a vision system can be used from road tracking, obstacle detection, or domain recognition. All these questions require the same initial step which consists in reducing the data contained in images. This step is called segmentation.

When a road does not include white lines, detection is a particularly difficult problem because contrasts are generally not strong enough to use an edge detection algorithm. In such case, images require the use of a region-based segmentation, where we are not looking for discontinuity, but, on the contrary, for homogeneous zones in the sense of one or several given criteria. Segmentation is achieved using a split and merge technique, and regions are represented by grey level and texture features. The originality of this algorithm is that texture is used only to improve on the results of the first detection using grey levels.

In the first part of this paper, the splitting step is presented which is achieved by studying a co-occurrence matrix shape. Merging will be explained in the second part. Markov Random Fields are used to model exploited fields, and the segmentation is optimally determined using the Iterated Conditional Modes (ICM). Experimental results will be presented on road images without white lines.

\section{Our Approach}

\subsection{Splitting}

The splitting algorithm described here is based on the use of co-occurrence matrix [5] defined by R. Haralick [4]. We consider a window of interest (or sector) containing three regions with uniform grey level $a, b, c$. The co-occurrence 
matrix, and its diagonal can be represented by Fig. 1, where point size is proportional to the number of transitions (grey level - grey level) present in the window of interest. We notice that each region is characterized by a peak of the co-occurrence matrix diagonal.
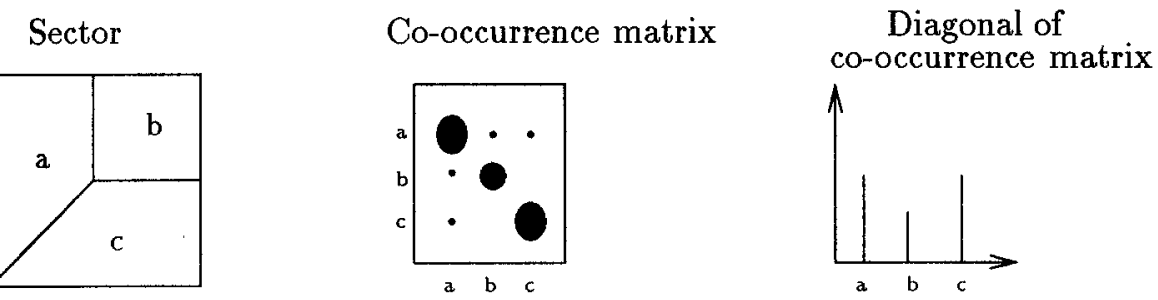

Fig. 1. Co-occurrence matrix

The splitting algorithm based on this particularity, can be broken down into five parts :

- Dividing the image into sectors : this is done independently of data, and consists in putting a grid on the image. We will discuss later the size of the grid elements.

- Computation of the co-occurrence matrix : for each sector, we compute the co-occurrence matrix diagonal. The matrix will be computed from a distance of one pixel, a neighborhood of eight pixels, and orientations of 0 , and 90 degrees.

- Peak extraction of the co-occurrence matrix diagonal : different classes of the diagonal are extracted, a class being defined as a peak and its two adjacent valleys.

- Image labeling: we create an image of labels in which different labels are given to each set of connected pixels, belonging to the same class. Such pixel sets are called regions.

- The removal of small regions : small, non-significant regions (whose number of pixels is lower than a threshold which we discuss later) are removed by merging them with the neighboring region which has the closest grey levels average.

The results of the splitting step on road images without white lines are shown in Fig. 2. The initial size of the sectors is $32 \times 32$ pixels, and we simply work with a frame. Thus, we find sectors of $32 \times 64$ pixels on the result images.

Figure 2 presents the splitting results for two images, each region being represented by its outlines. When two neighboring regions have very different grey levels, their common outline is very well detected, and the splitting results are 


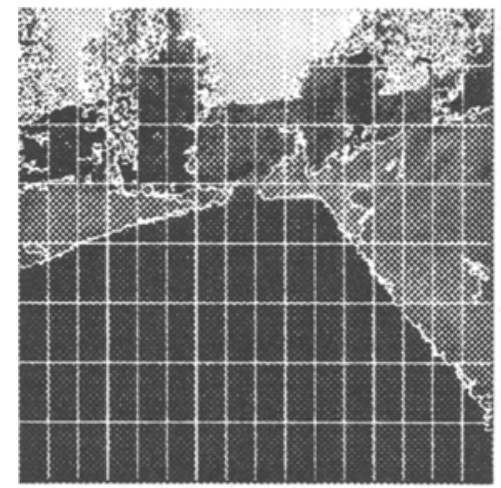

a - Splitting result on image 1

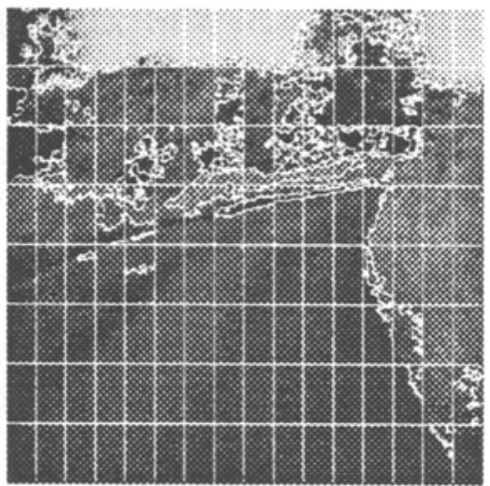

b - Splitting result on image 2

Fig. 2. Results.

sufficient to initiate the merging process. On the other hand, when some textured regions are presented, with a similar grey levels average, the splitting algorithm, which use only the diagonal of the co-occurrence matrix does not separate the two regions. So, we must use texture features in the segmentation algorithm. These features are only introduced during the merging step. The reasons for this choice are explained in the following section.

\subsection{Merging}

As seen above (see Fig. 2-b), it is not sufficient to use grey level data to obtain robust image segmentation algorithm (in order to find road edges). In this case, we will include texture parameters. Among all extraction methods of texture parameters, we have chosen to use texture features which are computed from the co-occurrence matrix [4], i.e. homogeneity and energy. Several authors such as M. Derras [2] have split original images using a grid, and computed texture parameters on sectors obtained in this way. The greatest disadvantage of this method is that it leads to a strong resolution.

For these reasons we compute texture coefficients from regions produced during the splitting step. Segmentation will then provide one pixel resolution edges, when the computed image presents homogeneous grey level. For textured images, resolution will be identical to the initial grid. Some initial sectors of $32 \times 32$ pixels are chosen. The strongest resolution will also be 32 pixels.

The final step of the splitting process is the removal of small regions. A small region is defined by a number of pixels lower than a threshold. This threshold is fixed at the minimal number of pixels to be considered for texture computation. Here, it is fixed at fifty pixels.

In order to obtain an autonomous merging algorithm, Markov Random Fields are used. They have been widely quoted in literature. D.W. Murray et B.F. Buxton [7] have obtained a spatio-temporal segmentation algorithm by mod- 
eling apparent motion with Markov Random Fields. H. Derin, et H. Elliot [1] and S. Geman et C. Graffigne [3] have obtained textured image segmentation algorithms. M. Derras [2] used Markov Random Fields to achieve natural UNsupervised image segmentation. We should also mention C. Kervrann et F. Heiz [6] who propose a segmentation method of textured images with a Markov relaxation.

Input data in our possession are regions produced by splitting. They are modeled by a features vector with four components : the grey levels average, the peak of the co-occurrence matrix diagonal, the homogeneity, and the energy. These regions cannot be split further and we call them elementary sites, considering them as data fields (denoted $\mathrm{Y}$ ). The problem is then to establish a correspondence between this data field, and a label field $\mathrm{X}$, for which a label represents a set of homogeneous regions. We model exploited fields using Markov Fields which will be integrated into a Bayesian formalism in order to use constraints derived from a priori knowledge.

The algorithm we present was inspired by the ICM (Iterated Conditional Mode) algorithm : at each iteration, all sites are observed, and, for each of them, we compute the different possible values of an a posteriori energy function with respect to different labels. Only the label with minimal energy function is retained for this site. An a posteriori energy function is modeled by :

$$
U(X \backslash Y)=U(Y \backslash X)+U(X) .
$$

Or, for a given site,

$$
U\left(X_{s} \backslash Y_{s}\right)=U\left(Y_{s} \backslash X_{s}\right)+U\left(X_{s}\right)
$$

where :

- $U\left(X_{s}\right)$ is the a priori energy function related to the label field. We also denote it $U\left(e_{s}\right)$

- $U\left(Y_{s} \backslash X_{s}\right)$ is the energy function representing the probability of observation, given the pixel label $X_{s}$. We denote it $U\left(O_{s} \backslash e_{s}\right)$.

With these new notations, we obtained :

$$
U\left(e_{s} \backslash O_{s}\right)=U\left(O_{s} \backslash e_{s}\right)+U\left(e_{s}\right) .
$$

Then, we defined a neighborhood system $V=\left\{V_{s}, s \in S\right\}$ on $S$ such that $s_{1} \in V_{s 2}$ if, and only if, the sites $s_{1}$ and $s_{2}$ are adjacent in the sense of 8-connexity.

We model an a priori energy function $U\left(e_{s}\right)$ so that two neighboring sites will probably belong to the same label. The energy function can be noted :

$$
U\left(e_{s}\right)=\sum_{r \in V_{s}} V_{c}\left(e_{r}\right) .
$$

where $V_{c}$ is the energy potential so that :

$$
\begin{aligned}
V_{c}\left(e_{s}\right) & =0 \text { if } e_{s}=e_{t} . \\
& =1 \text { else } .
\end{aligned}
$$


where $e_{t}$ is the second site of the clique $c$. Here, a clique is a set of two elements : the considered site, and one of the neighbor sites.

The transposition energy will be modeled by the Euclidean distance between feature vectors of the site $s$, and those of the label $e$ :

$U\left(O_{s} \backslash e_{s}\right)=\left(m o y_{s}-\text { moy }_{e}\right)^{2}+\left(\text { pic }_{s}-p i c_{e}\right)^{2}+\left(\text { hom }_{s}-\text { hom }_{e}\right)^{2}+\left(e n e_{s}-e n e_{e}\right)^{2}$

\section{Experimental Results}

We present here, segmentation results obtained from the previously used images (Fig. 3-a and 3-b).

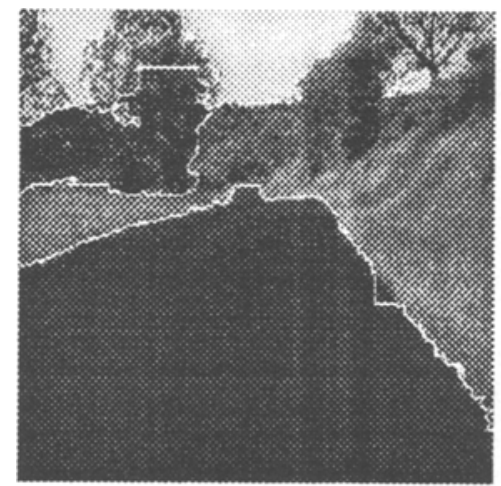

a- Segmentation result on image 1

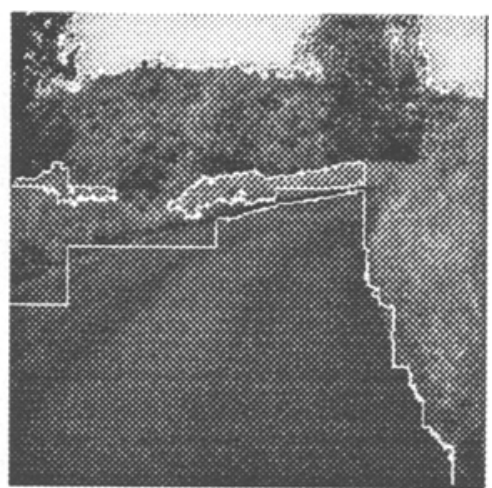

b- Segmentation result on image 2

Fig. 3. Results.

Generally, the road area is well detected. In the worst case, the resolution is the same as in the initial grid. So the segmentation algorithm provides elements which are necessary to a possible interpretation process. For some $512 \times 512$ images, this segmentation algorithm takes approximately 2.5 seconds on a HP9000/735.

\section{Conclusion and Perspectives}

The aim of this work is to segment road images without white lines. We were confronted with a difficulty which is that grey levels were not sufficient in these images, so we decided to use texture. We also abandoned all methods based on edge detection and instead we used a region-based segmentation algorithm. Our first approach was to divide images into sectors, and to compute texture on each 
sector. A disadvantage of this method is that the resolution of the segmented image is that of the initial grid. On the one hand we have segmentations using grey level whose resolution is only a one pixel resolution, but the road area cannot be found in some images. On the other hand we have segmentations using texture, which finds the road area, but has a high resolution. So, we decided to use both of this two major class algorithm. First, we split the images using a homogeneity criterion based on grey levels. Then, we compute texture features on the resulting regions. Both parameters (texture and grey level) are used to achieve merging. This segmentation algorithm provides results which have a one pixel resolution when grey levels are sufficient to extract the road area. When the road area and the background have the same grey levels average, and different textures, resolution is the same than in initial grid. This algorithm, which is entirely UN-supervised, gives very satisfying results for images which do not include objects which are too small relative to the initial grid size. It can be applied both on textured images and on UN-textured images. Furthermore, the segmentation algorithm provides elements which are necessary to a possible interpretation process.

\section{References}

1. Derin, H.,Elliot, H.: Modeling and Segmenting of Noisy and Textured Images using Gibbs Random Fields. IEEE trans. on pattern analysis and machine intelligence PAMI 9 n 1 (1987)

2. DERRAS, M.: Segmentation non Supervisée d'Images Texturées par Champs de Markov : Application à l'Automatisation de l'Entretien des Espaces Naturels. Thèse de docteur es Sciences LASMEA Clermont-Ferrand (dec. 1993) $174 \mathrm{p}$

3. GEMAN, S.,GRAFFIGNE, C.: Markov random fields image and their applications to computer vision. Proceeding of the International Congress of Mathematicians Ed. A.M. Gleason American Mathematical Society Providence (1987)

4. HARALICK, R., SHANMUGAN, K.,DINSTEIN, H.: Textural Features for Image Classification. IEEE Transactions on Systems and cybernetics vol. SMC-3 (1973) 610-621

5. HOUZELLE, S.,GIRAUDON, G.: Segmentation Région par Modélisation de Matrices de Cooccurrence. AFCET $8^{\text {eme }}$ congrès Reconnaissance des formes et intelligence artificielle Lyon Villeurbanne vol 3 (Nov 1991)

6. KERVRANN, C.,HEITZ, F.: A Markov Random Field Model-Based Approach to Unsupervised Texture Segmentation Using Local and Global Spatial Statistics. INRIA n 2062 (1993)

7. MURRAY, D.W.,DUXTON, B.F.: Scene Segmentation from Visual Motion using Global Optimization. IEEE transactions on pattern analysis and machine intelligence vol PAMI 9 n 2 (1987) 220-228 